\title{
The vibration characteristics of the transformer winding calculation and experimental research
}

\author{
Yaning $\mathrm{Li}^{1,2}$, a , Jun Wang ${ }^{1}$, Hong $\mathrm{Yu}^{3}$, Guochao Qian ${ }^{3}$ and Xiaoyan $\mathrm{Zhu}^{1}$ \\ ${ }^{1}$ North China Electric Power University, Baoding, China \\ ${ }^{2}$ North China Electric Power University graduate student workstation of Yunnan Power Grid Corporation, Kunming, \\ China; \\ ${ }^{3}$ Yunnan Power Grid Electric Power Research Institute Co., Ltd., Kunming, China
}

\begin{abstract}
Winding vibration electric power transformer is under circulation in the leakage magnetic field generated by currents in the windings due, calculated winding paper analyzes the distribution of electric power to analyze the vibration characteristics of the windings. Using finite element analysis software ANSYS finite element solid modeling transformers, electric power distribution and obtain model vibration response at electric power stimulation, with high efficiency and accuracy. The results shows that the calculated results are in good agreement with the experimental results illustrate the correctness of the calculation results.
\end{abstract}

Keywords: transformer; winding vibration; finite element modeling; electric power.

\section{Introduction}

Transformer is one of the more expensive and important equipment in power system. Its stability and reliability are related to the security of the whole power network. If a large power transformer in the operation of the system has an accident, it may cause a large area power outages, and its maintenance period is generally six months or more, not only a huge, but also a very wide impact [1]. In the transformer operation accident, the winding related failures accounted for nearly half of the total number of faults, from home and abroad, the statistics show that the vibration of the winding is one of the reasons for the failure of the transformer [2-4]. The current carrying conductor of the winding coil of the transformer is in the magnetic field, and the electric power is on the conductor. Electric power in the transformer coil material to produce mechanical stress, and so that the winding vibration, part of the transfer to other components of the transformer. Under the rated current, the electric power is not great, but in the case of short circuit, the electric power increases sharply, when the power of the winding is destroyed, the power of the transformer will collapse.

Transformer winding vibration is caused by the electric power generated by the flow of the leakage magnetic field. Therefore, it is very important to analyze the electric power distribution. In this paper, the electromagnetic field and vibration of the transformer windings are analyzed, and the advantages of the finite element analysis software ANSYS in solid modeling are used to establish the three-dimensional solid model of the transformer. After dividing the model, the electromagnetic excitation force is applied, and the magnetic field of the winding leakage, the electric power

a Corresponding author : HDliyaning@163.com

(C) 2016. The authors - Published by Atlantis Press 


\section{AEST2016}

distribution and the axial electric power distribution of the transformer windings are obtained. And the results are compared with the experimental results, the results are in good agreement, which shows the correctness of the results.

\section{Basic principle}

First set up electromagnetic analysis problem solving area $V$ as shown in Figure $1, V_{1}$ is vortex area and iscontaining conductive medium but no source current; $V_{2}$ is not vortex area and is notcontaining conductive medium; $S_{12}$ is the internal interface between $V_{1}$ and $V_{2}$. The outer boundary $S$ is divided into two parts: $S_{B}$ and $S_{H}$.

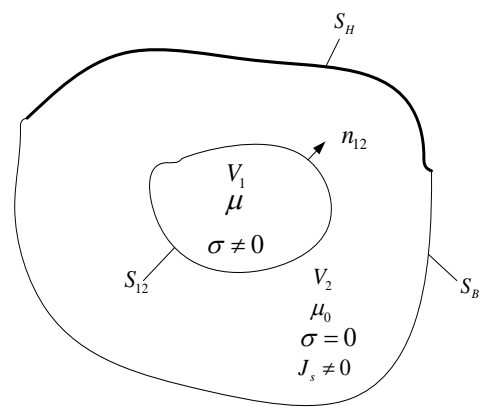

Figure 1. Layout of the electromagnetic field solver

Within the entire solution region $V$, field equations and boundary conditions are as follows: In $V_{1}$ :

$$
\left\{\begin{array}{c}
\nabla \times H=\sigma E \\
\nabla \times E+\frac{\partial B}{\partial t}=0 \\
\nabla \cdot B=0
\end{array}\right.
$$

$\operatorname{In} V_{2}$ :

$$
\begin{gathered}
\nabla \cdot B=0 \nabla \times H=J_{s} \\
\text { On } S_{B}: B \cdot n=0 \\
\text { On } S_{H}: H \times n=0 \\
\text { On } S_{12}:\left\{\begin{array}{c}
n_{12} \cdot B_{1}=n_{12} \cdot B_{2} \\
H_{1} \times n_{12}=H_{2} \times n_{12}
\end{array}\right.
\end{gathered}
$$

The general synthesis of electromagnetic field equations is as follows:

$$
[K]\left\{A_{\varphi}\right\}+[M] \frac{\partial}{\partial t}\left\{A_{\varphi}\right\}=[C]\{I\}
$$


If the winding consists of $n$ lines, in order to calculate the electric power that the winding can be subjected to each line of the winding, the winding can be divided into an $n$ area.The resultant force of each region is the electric power of the corresponding line. In the finite element model, the winding area has been dispersed. The electromagnetic field can be obtained from Eq.(6).Under the premise of solving the magnetic field distribution, according to the principle of Lorenz, for the discrete element $e$ :

$$
F^{e}=J^{e} \times B^{e} V^{e}
$$

According to the magnetic field distribution, the electric power distribution of the windings can be calculated, as follows:

$$
F^{e}=J^{e} \times B^{e} V^{e}=V^{e}\left|\begin{array}{ccc}
i & j & k \\
J_{x}^{e} & J_{y}^{e} & J_{z}^{e} \\
B_{x}^{e} & B_{y}^{e} & B_{z}^{e}
\end{array}\right|
$$

Omit the $z$ direction, calculation formula of two-dimensional distribution of electromagnetic force is obtained.

Axial vibration equation of winding under electromagnetic force as follows:

$$
\left[M_{0 Y}\right] \frac{d^{2}\{Y\}}{d t^{2}}+\left[C_{0 Y}\right] \frac{d\{Y\}}{d t}+\left[K_{0 Y}\right]\{Y\}=\left\{F_{Y}\right\}+\left\{m_{0}\right\} g
$$

And then, the axial displacement of each solution can be obtained by changing the axial displacement of the two time derivative, and the acceleration of the axial vibration of the coil can be obtained.

\section{Finite element modeling}

Using the finite element analysis software ANSYS, the process of 3D solid modeling and solving of the transformer windings is as follows:

a) According to the geometrical size of the high voltage winding of transformer, the 3D solid model is used to establish the model of winding geometry.

b) Set winding and insulation cushion block of material properties, including winding and the cushion block unit density, Poisson's ratio and elastic modulus value.

c) The geometry model is reasonable, and the freedom degree constraint is set.

d) Finite element modal analysis is carried out to obtain the natural frequency and the corresponding mode of the winding.

e) The calculation results are compared with the experimental results, and the model is modified according to the experimental results.

Using the finite element software ANSYS, the 3D solid modeling of the transformer windings is carried out. The large transformer winding is a pie type laminated structure, and the solid model of the high voltage winding of the transformer is carried out according to the experiment, as shown in Fig.2. 


\section{AEST2016}

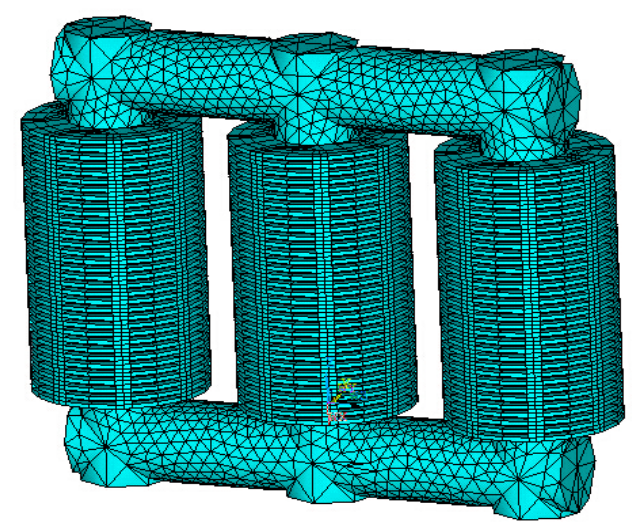

Figure 2. Three-dimensional finite element model of the transformer windings

\section{Result analysis}

The electromagnetic transient analysis is used in the calculation, and the excitation source is $50 \mathrm{~Hz}$ current density. The calculation step is $0.001 \mathrm{~s}$, and the 100 steps are calculated. The electromagnetic field distribution of 0.008s in the electromagnetic field is shown in Figure 3. The reason for this is that when the $8 \mathrm{~ms}$ is in the transformer core, the magnetic flux is the largest, which can reflect the distribution of the magnetic field.

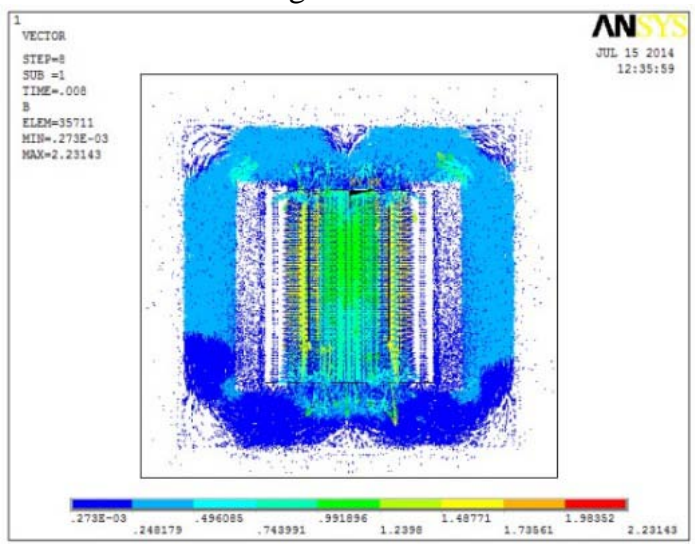

Figure 3. Leakage Field

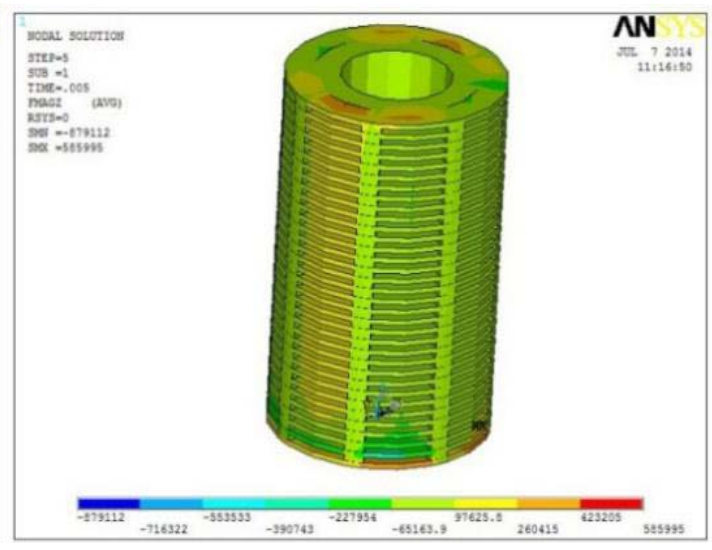

Figure 4. Electric power distribution

Power distribution is shown in Figure 4. The axial electromagnetic force of the transformer winding is not uniform, and the electromagnetic force on the same line is different from that in the same line. 


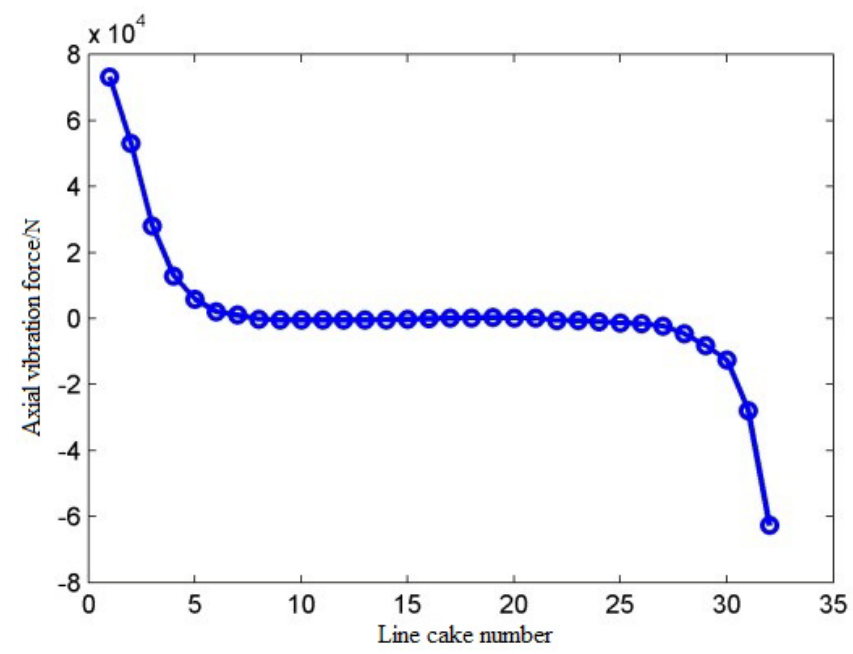

Figure 5. Axial electric power distribution transformer windings

The power distribution of the transformer windings is shown in Figure 5. The power of the transformer windings is presented in the middle of the winding, which is distributed in the center of the winding.

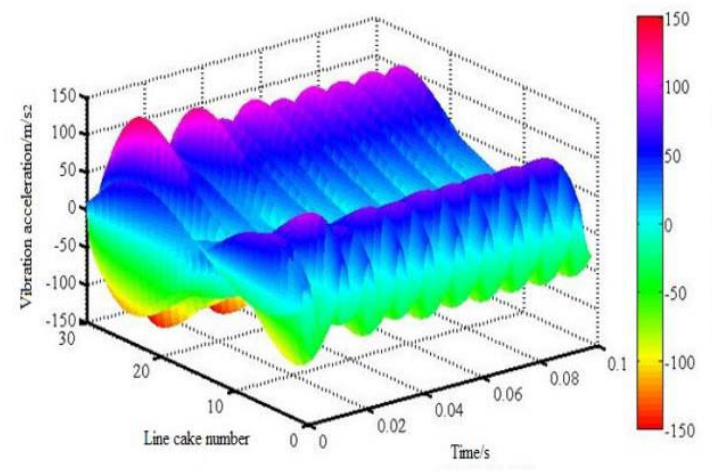

(a)

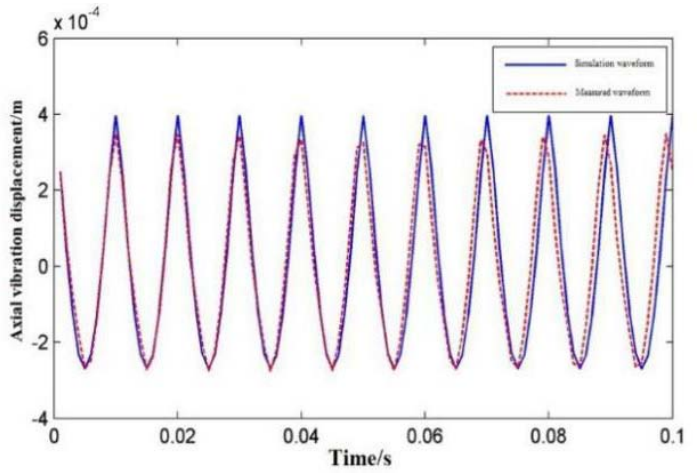

(b)

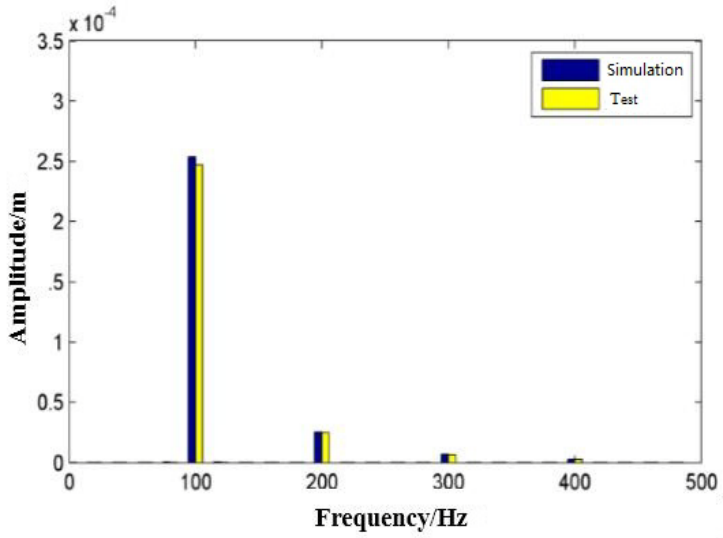

(c)

Figure 6. Vibration characteristics of the transformer (a) winding vibration characteristics (b)vibration wave and (c) Frequency spectrum of vibration wave

Calculation of vibration characteristics of transformer windings looks as follows:Figure 6. The 
vibration amplitude of the transformer windings is $100 \mathrm{~Hz}$ component, and the vibration amplitude of the line is different. Similar to the electric power distribution, the vibration amplitude in the middle of the line is the smallest. In addition, the displacement and acceleration of each line of the cake show the vibration and fluctuation, and the maximum and minimum values are reached in the order of the serial numbers, and the maximum and minimum values are not the same. In order to verify the correctness of the calculation results, the calculated results are compared with the measured results. The results are compared with the measured results, and the results of the measurement results are obtained directly by the fiber optic vibration sensor. Obviously, the calculation results are in good agreement with the measured results, which shows the correctness of the results.

\section{Conclusions}

The electromagnetic field and vibration of the transformer windings are calculated, and the simulation results are compared with the experimental results by using ANSYS finite element modeling:

a) The simulation results are in agreement with the measured results, which show the accuracy of the calculation of the vibration characteristics of the transformer windings;

b) The vibration response of transformer windings is $100 \mathrm{~Hz}$, and the amplitude of each line is different;

c) The vibration amplitude is the smallest in the middle of the electric power distribution, and the displacement and acceleration of each line are shown in the vibration and wave.

\section{References}

1. He Y Y. Discussion on Short Circuit Capacity of Power Transformer. Transformer, 33(9) :2-7, (1996)

2. Li J. The Trouble Pattern and Reliability Operation Small- Medium Size Power Transformers. Transformer, 34(4): 9-12, (1997)

3. Wang M Y, Ling M. Statistics and analysis on short-circuit faults of large power transformers [J]. Trasformer, 34(10): 12-17, (1997)

4. Jin W L, Chen J H, Li Y M, etal. Statistics and Analysis on Power Transformer Damages Caused by Short-Circuit Fault in $110 \mathrm{kV}$ and Higher Voltage Classes. Power System Technology, 23(6): 21-25, (1999)

5. Guo J, Lin H Y, Xu Z H, etal. Analysis of axial stability of power transformation windings using finite element [J]. High Voltage Engineering, 33(11): 209-212, (2007)

6. Garcia B, Burgos J C, Alonso A M. Transformer tank vibration modeling as a method of detecting winding deformations-part I: theoretical foundation [J]. IEEE Transactions on Power Delivery, 21(1): 157-163, (2006)

7. L. F. Shampine. Implementation of implicit formulas for the solution of ODEs [J]. SIAM J. Sci. and Stat. Compute.. 1(1):103-118(1980)

8. Shao Y Y. Theoretical and experimental study of vibration features of large-scale power transformer [D]. Master Dissertation of Shanghai Jiaotong University, (2006).

9. Garcia B, Burgos, J.C, Alonso, A.M. Transformer tank vibration modelling as a method of detecting winding deformations-part I: theoretical foundation., IEEE Transactions on Power Delivery, 21(1): 157-163, (2006)

10. Garcia B., Burgos, J.C, Alonso, A.M. Transformer tank vibration modelling as a method of detecting winding deformations-part II: experimental verification. IEEE Transactions on Power Delivery, 21(1),164-169, (2006)

11. Fu J, Shao Y Y. The experiment of diagnosing large-scale transformer winding looseness by frequency response analysis [J], East China Electric Power, 34(4): 28-29, (2006)

12. $\mathrm{Li} \mathrm{H} \mathrm{K,} \mathrm{Li} \mathrm{Y.} \mathrm{Axial} \mathrm{vibration} \mathrm{modal} \mathrm{analysis} \mathrm{of} \mathrm{transformer} \mathrm{windings} \mathrm{under} \mathrm{different} \mathrm{level} \mathrm{of}$ pre-compression[J]. Electric Machines and Control, 14(8): 98-101l, (2010). 